Biochemical Pharmacology, 1966, Vol. 15, pp. 396-399. Pergamon Press Ltd., Printed in Great Britain.

\title{
The inhibition of cerebral glutamine synthetase by structural analogs of the convulsant $d l$-methionine sulfoximine
}

(Received 5 October 1965; accepted 5 November 1965)

GLUTAMINE synthetase [L-glutamate-ammonia ligase (ADP)] (EC 6.3.1.2) of rat cerebral cortex may be readily inhibited by the convulsant agent $d l$-methionine-dl-sulfoximine (MSO), both in vitro and in vivo. ${ }^{1,2}$ Although in vitro this inhibition is readily reversible by excess substrate, L-glutamate, the inhibition in vivo appears to reflect a nonreversible interaction of the convulsant drug with the enzyme protein, which becomes maximal at the time the animal experiences the first seizure episode and which, as has been suggested, ${ }^{2}$ possibly involves conformational changes in the enzyme molecule. As neither methionine sulfoxide nor methionine sulfone could be shown to inhibit in vivo the cerebral glutamine synthetase-glutamine transferase system, ${ }^{2}$ the examination of the contribution of the sulfoximinomoiety of MSO as a determining factor conferring specificity to the drug-enzyme interaction became of interest.

In this communication we wish to report results of experiments in which four structural analogs of MSO, three S-alkyl-homocysteine sulfoximines, and one S-alkyl cysteine sulfoximine were evaluated as inhibitors of cerebral glutamine synthetase in vitro.

\section{MATERIALS AND METHODS}

Rat cerebral cortex was homogenized in ice-cold distilled water. ${ }^{3,4}$ and the homogenate (about $1: 10, \mathrm{w} / \mathrm{v}$ ) was centrifuged at $11,000 \mathrm{rev} / \mathrm{min}$ in a Servall SS-34 superspeed centrifuge. The resulting sediment was resuspended in cold distilled water and recentrifuged as above and the supernatant added to that obtained after the first centrifugation. Sufficient $1 \mathrm{M} \mathrm{NaCl}$ was then added to make the pooled supernatants $0.2 \mathrm{M}$ in salt, and the solution was centrifuged at 17,000 rev/min for $1 \mathrm{hr}$. The resulting supernatant was used as the source of enzyme. Glutamine synthetase activity was routinely determined in a system containing in $3 \mathrm{ml}$ : K-glutamate, $20 \mathrm{mM}$; Tris-HCl buffer, pH 7.2, $80 \mathrm{mM}$; $\mathrm{Mg}^{2+}, 20 \mathrm{mM}$; freshly neutralized $\mathrm{NH}_{2} \mathrm{OH}-\mathrm{HCl}, 50 \mathrm{mM}$; ATP (Na salt), 13.3 mM; BAL, $0.5 \mathrm{mM}$; and suitable volumes of enzyme. After incubation ( $30 \mathrm{~min}, 37^{\circ}$ ) and enzyme inactivation, the assay was carried out as previously described. ${ }^{3}$ In the control tubes, ATP was omitted. Inhibitor was added in water solution at concentrations indicated below.

The following newly synthesized compounds were tested: S-ethyl-dl-homocysteine sulfoximine (ethionine sulfoximine, ESO), S-ethyl-dl-cysteine sulfoximine (ECSO), S- $n$-propyl-dl-homocysteine sulfoximine (PSO), and S-n-butyl-dl-homocysteine sulfoximine (BSO).*

\section{A. S-ethyl-dl-homocysteine sulfoximine (ESO)}

S-methyl-dl-homocysteine-dl-sulfoximine, MSO, has been previously found to inhibit glutamine synthetase competitively with respect to one of its substrates, viz. L-glutamate. ${ }^{1}$ Thus, $5 \times 10^{-4} \mathrm{M}$ MSO inhibits the enzyme 100,80 , and 40 per cent at concentrations of L-glutamate of $4 \times 10^{-4} \mathrm{M}$, $4 \times 10^{-3} \mathrm{M}$, and $4 \times 10^{-2} \mathrm{M}$ respectively, As shown in Fig. 1, the lowest concentration of ESO effective as an inhibitor was $1 \times 10^{-3} \mathrm{M}$, an inhibition of 20 per cent resulting at a concentration of L-glutamate of $1.6 \times 10^{-3} \mathrm{M}$. Raising [I] to $13 \times 10^{-3} \mathrm{M}$, while keeping [S] at $1.6 \times 10^{-3} \mathrm{M}$ increased the inhibition to about 80 per cent. When [S] was increased to $10 \times 10^{-3} \mathrm{M}$, inhibition was about 50 per cent at $13 \times 10^{-3} \mathrm{M}$ inhibitor, i.e. with a ratio of substrate to inhibitor of approximately 1. It could be calculated that for [S]: $10 \times 10^{-3} \mathrm{M}$, MSO at a concentration of about $1 \times 10^{-3} \mathrm{M}$ was sufficient to inhibit the synthetase by about 50 per cent, indicating a more than 10 -fold greater inhibitory capacity of MSO as compared to ESO under these conditions. Plots of [I] (ESO) vs. 1/v

* The synthesis, characterization, and study of the chromatographic behavior of the new sulfoximines and of several sulfoxides of S-alkyl cysteines and homocysteines will be described elsewhere (O. Z. Sellinger and Margaret A, Clarke). 
at two substrate concentrations and of $1 / \mathrm{S}$ vs. $1 / \mathrm{v}$ at $[\mathrm{I}]: 9 \times 10^{-3} \mathrm{M}$ gave evidence of competitive inhibition. A $K_{i}$ value of $3.2 \times 10^{-3} \mathrm{M}$ was calculated.

\section{B. S-ethyl-dl-cysteine sulfoximine (ECSO)}

At equimolar concentrations and with $\mathrm{L}$-glutamate at $10 \times 10^{-3} \mathrm{M}$, this analog of MSO was found to be equally effective as an inhibitor of glutamine synthetase as ESO (Table 1). However, while inhibition by ESO became proportionately more severe as the concentration of L-glutamate decreased or, conversely, that of inhibitor increased (Fig. 1), inhibition by ECSO appeared to be less dependent on substrate concentration and failed to exceed a 50 per cent inhibition plateau.

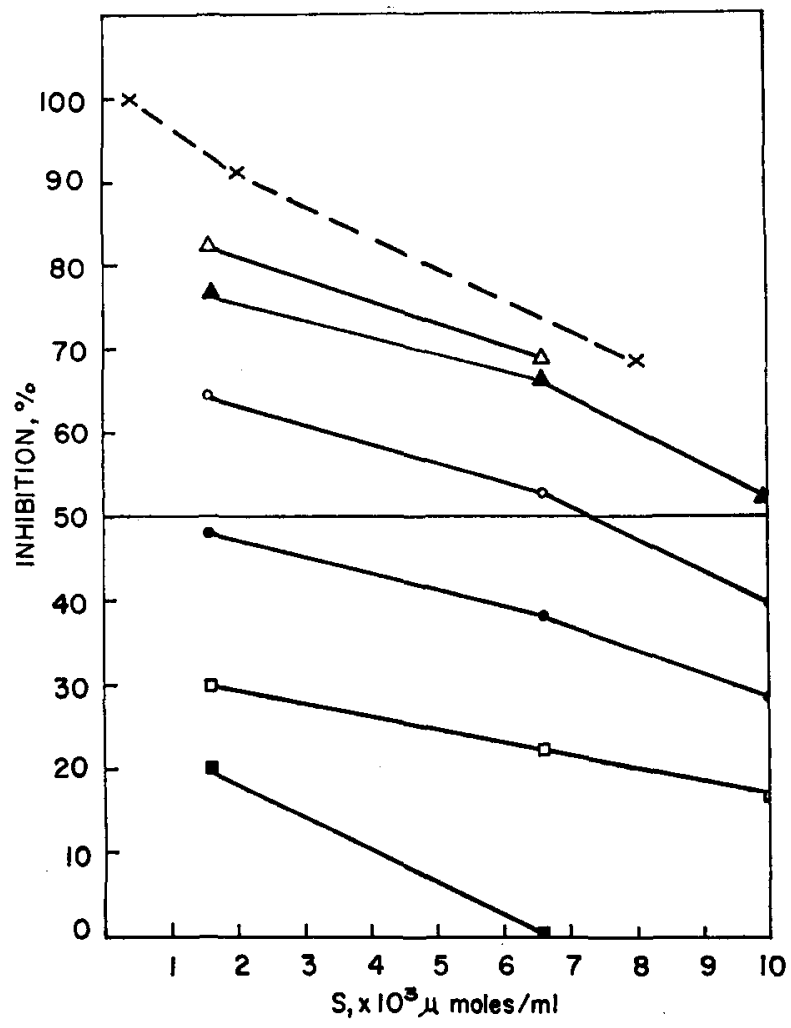

FIG. 1. The inhibition of cerebral glutamine synthetase by S-ethyl-dl-homocysteine-dl-sulfoximine (ESO): the effect of varying the concentration of substrate, L-glutamate. The per cent inhibition values were determined in vitro in an assay system, as described in the text. Inhibitor was added to the following final concentrations: $\square-1 \times 10^{-3} \mathrm{M} ; \square-\square, 2 \times 10^{-3} \mathrm{M} ; \mathrm{O}-4.8 \times 10^{-3}$ $\mathrm{M} ; \mathrm{O} \longrightarrow \mathrm{O}, 9 \times 10^{-3} \mathrm{M} ; \Delta \sim \Delta, 13 \times 10^{-3} \mathrm{M} ;$ and $\Delta-\Delta, 14 \times 10^{-3} \mathrm{M}$. The dashed line represents the inhibition of glutamine synthetase in the presence of $5 \times 10^{-4} \mathrm{M}$ methionine sulfoximine.

\section{C. $S$-n-propyl-dl-homocysteine sulfoximine (PSO)}

This MSO analog was found to be less effective as a glutamine synthetase inhibitor than either ESO or ECSO (Table 1). This was particularly evident in the range of inhibitor concentrations between 9 and $14 \mathrm{mM}$ where approximately 50 per cent less inhibition was obtained as compared to ESO.

\section{D. $S$-n-butyl-dl-homocysteine sulfoximine (BSO)}

Lengthening the S-alkyl side chain from 3 to 4 carbons resulted in a further drop of the inhibitory power (Table 1). Thus, $2 \mathrm{mM}$ BSO produced no inhibition, and $14 \mathrm{mM}$ BSO was only half as effective as the same concentration of ESO. 
TABLE 1. INHIBITION OF CEREBRAL GLUTAMINE SYNTHETASE BY ANALOGS OF METHIONINE SULFOXIMINE*

\begin{tabular}{ccccc}
\hline \multirow{2}{*}{$\begin{array}{c}\text { Concentration of } \\
\text { analog } \\
(\mathrm{mM})\end{array}$} & ECSO & ESO $\dagger$ & PSO & BSO \\
\cline { 2 - 5 } & 21 & 17 & 14 & 0 \\
2 & 43 & 40 & 18 & 10 \\
9 & 48 & 55 & 32 & 25 \\
14 & 51 & & 45 & 43 \\
28 & & & &
\end{tabular}

\footnotetext{
* For comparison, $5 \times 10^{-4} \mathrm{M}$ MSO (glutamate, $8 \mathrm{mM}$ ) inhibits 68 per cent. ${ }^{1}$

Concentration of L-glutamate was $10 \mathrm{mM}$. The assay system, as described in Materials and Methods, was used.

† Calculated from data in Fig. 1.
}

\title{
DISCUSSION
}

The structural requirements for successful inhibition of cerebral glutamine synthetase by substances competing with and resembling one of its substrates, viz. L-glutamate, have not been studied in detail. Braganca et al. ${ }^{5}$ observed that MSO as well as methionine and ethionine sulfoxide may successfully relieve the inhibition of synthesis of bound acetylcholine produced in a brain-slice system by the exogenous addition of ammonium ions. Other reports ${ }^{\theta-11}$ have described an inhibitory action upon bacterial and sheep brain glutamine synthetase of methionine sulfoxide and of MSO, but only the latter molecule has been effective in inhibiting glutamine synthetase from rat cerebral cortex ${ }^{2}$ both in vitro and in vivo. S-Benzyl homocysteine sulfoxide has also been reported to inhibit the growth of Lactobacillus arabinosus, an event which fails to take place in the presence of glutamine and which is reversed by increased levels of glutamate. ${ }^{12}$ Similar observations of Escherichia coli have been made with 3-amino-3-carboxypropanesulfonamide, the sulfonamide analog of glutamine. ${ }^{13}$ More recently, Mastalerz $^{14}$ has found $\gamma$-phosphonoglutamate and P-ethyl- $\gamma$-phosphonoglutamate to be strong inhibitors of glutamine synthetase.

The present finding that the elongation of the S-alkyl side chain in the homocysteine sulfoximine series from $\mathrm{CH}_{3}$ to $n$-butyl results in a gradual decline of the inhibitory power of the corresponding sulfoximine, indicates that substitution of the $S$ atom and, to a lesser extent, the overall length of the inhibitor molecule are of importance as determinants of the enzyme-inhibitor interaction. Thus, in view of the equal inhibitory power of the S-ethyl-substituted sulfoximines, ECSO and ESO, a 5- and a 6-carbon compound, respectively, it would appear that the length of the alkyl substituent on the $\mathbf{S}$ atom, rather than the total length of the molecule, determines the effectiveness of the drug-enzyme interaction in vitro. Insofar as the action in vivo of these MSO analogs as convulsants is concerned, the intracerebral administration of ESO to rats has resulted in convulsions; however, the effective dose was about 100 times as large as that required with MSO.

The data presented indicate that the structural analogs of MSO tested so far are considerably less effective as competitive inhibitors of cerebral glutamine synthetase than is MSO itself. Strict structural requirements appear to exist for the competitive binding of the sulfoximines to the glutamine synthetase protein, one of which is the presence of the S-methyl group. The modalities governing this type of interaction, as well as the irreversible binding of MSO to glutamine synthetase which results in permanent activity loss, are at present under investigation.

\author{
Mental Health Research Institute, \\ O. Z. Sellinger* \\ University of Michigan, \\ Aura Garaza $\dagger$
}

Ann Arbor, Mich., U.S.A.

* Research supported by Grant NB-04549-02 from the U.S. Public Health Service. Work done as a visiting Fulbright Professor, Dept. of Biochemistry, School of Medicine, University of the Republic, Montevideo, Uruguay.

$\dagger$ Miss Garaza's present address: School of Chemistry, University of the Republic, Montevideo, Uruguay. 


\section{REFERENCES}

1. O. Z. Sellinger and P. G. Weiler, JR., Biochem. Pharmac. 12, 989 (1963).

2. C. LAMAR, JR. and O. Z. Sellinger, Biochem. Pharmac. 14, 489 (1965).

3. O. Z. Sellinger and F. de Balbian Verster, J. biol. Chem. 237, 2836 (1962).

4. F. De Balbian Verster, O. Z. Sellinger and J. C. Harkin, J. cell. Biol. 25, 69 (1965).

5. B. M. Braganca, P. Faulkner and J. H. Quastel, Biochim. biophys. Acta 10, 83 (1953).

6. J. PACE and E. E. MCDermotr, Nature, Lond. 169, 415 (1952).

7. H. WAelsch, in Biochemistry of the Central Nervous System, Ed. F. Brucke, Pergamon Press, London (1959).

8. W. H. ElliotT and E. F. GALE, Nature, Lond. 161, 129 (1948).

9. E. Borek, H. K. Miller, P. Sheiness and H. Waelsch, J. biol. Chem. 163, 347 (1946).

10. J. G. HeathCote and J. PACE, Nature, Lond. 166, 353 (1950).

11. J. F. SPECK, J. biol. Chem. 179, 1405 (1949).

12. H. Waelsch, P. Owades, H. K. Miller and E. Borek, J. biol. Chem. 166, 273 (1946).

13. D. B. ReISNER, J. Am. chem. Soc. 78, 5102 (1956).

14. P. Mastalerz, Archs Immunol. Ter. dosw. 7, 201 (1959); Chem. Abstr. 54, 6843b (1960).

Biochemical Pharmacology, 1966, Vol. 15, pp. 399-400. Pergamon Press Ltd., Printed in Great Britain.

\section{Inhibition of intestinal amino acid transport by dimethyl sulfoxide*}

\section{(Received 11 October 1965; accepted 12 November 1965)}

TREATMENT of the malabsorptive state which is due to intestinal lesions is at present unsatisfactory. We have therefore begun a series of studies to evaluate the effect of agents that might increase the intestinal absorption of nutrients. One of the compounds tested, dimethyl sulfoxide (DMSO), had previously been utilized both as a vehicle for other compounds and as an anti-inflammatory agent because of its solvent and penetrant abilities. ${ }^{1,2}$ It has been noted that DMSO has a low order of systemic toxicity, but that it produces local toxic actions at high concentrations. ${ }^{3}$ We wish to report that one of these undesirable effects, at high concentrations of DMSO, is inhibition of active amino acid transport. This raises doubts whether large quantities of DMSO could be used as a solvent to improve nutrient entry from the intestine in cases of malabsorption.

Transport of $1 \times 10^{-4} \mathrm{M}$ L-alanine was followed by means of the generally labeled ${ }^{14} \mathrm{C}$ compound (New England Nuclear Corp.). Everted intestinal sacs were prepared from adult hamsters of both sexes, which were allowed food and water ad libitum, ${ }^{4}$ The initial condition was the same concentration of L-alanine in both mucosal $(5 \mathrm{ml})$ and serosal $(1 \mathrm{ml})$ solutions. After gassing with $95 \% \mathrm{O}_{2}+$ $5 \% \mathrm{CO}_{2}$, flasks were stoppered and incubated for $1 \mathrm{hr}$ at $37^{\circ}$. Mucosal and serosal fluids were drained, centrifuged to remove sloughed tissue, and then assayed for radioactivity. Transport was expressed in terms of micromoles L-alanine gained in the serosal fluid per unit wet weight of tissue. All values were compared with that of a control (three sacs from each of three hamsters, with Krebsbicarbonate buffer, $\mathrm{pH} 7 \cdot 4$, as the solvent). Various volumes of DMSO were used to replace the buffer, to produce concentrations up to approximately $5 \mathrm{M}$. The $\mathrm{pH}$ of such solutions was adjusted to $7 \cdot 4$ with a radiometer $\mathrm{pH}$ meter (although, because of nonaqueous DMSO effects, the actual $\mathrm{pH}$ may have been fractionally off this value; therefore, experiments were also run with the $\mathrm{pH}$ adjusted to 0.3 unit above and 0.3 unit below this value). Experiments were additionally performed with the buffer plus DMSO in which salts were added to readjust their concentration to that found in the original Krebs-bicarbonate buffer. Results of the transport experiments in these cases were identical with those in which the salt content was not readjusted (and slight variations in $\mathrm{pH}$ had no effect). Transport of L-alanine (nine sacs per experiment) was $52 \pm 10 \mathrm{~m} \mu \mathrm{moles} / 1.0 \mathrm{~g}$ sac (mean \pm S.D.) in the controls. There was no significant interference with transport at DMSO concentrations below $0.3 \mathrm{M}$. 